\title{
Stainless Steel Mesh Supported Carbon Nanofibers for Electrode in Bioelectrochemical System
}

\author{
Jing Wang, ${ }^{1}$ Ming Li, ${ }^{1}$ Fangtai Liu, ${ }^{2}$ and Shuiliang Chen ${ }^{1}$ \\ ${ }^{1}$ Department of Chemistry and Chemical Engineering, Jiangxi Normal University, Nanchang 330022, China \\ ${ }^{2}$ Institute for Advanced Study, Nanchang University, Nanchang 330031, China \\ Correspondence should be addressed to Shuiliang Chen; shuiliangchen@163.com
}

Received 22 October 2015; Revised 17 December 2015; Accepted 22 December 2015

Academic Editor: Vincenzo Baglio

Copyright (c) 2016 Jing Wang et al. This is an open access article distributed under the Creative Commons Attribution License, which permits unrestricted use, distribution, and reproduction in any medium, provided the original work is properly cited.

\begin{abstract}
We proposed a self-connected carbon nanofiber design for electrode in microbial bioelectrochemical system. This design was realized by direct growth of carbon nanofibers (CNFs) onto stainless steel (SSM) via a chemical vapor deposition process without addition of any external catalysts. In the CNFs-SSM composite electrode, the SSM acted as the conductive network and ensured efficient substrate and proton transfer, and the CNFs layer served as highly porous habitats for thick biofilm propagation. The current generated by the CNFs-SSM was 200 times higher than the bare SSM under the same experimental conditions. This provided a simple and promising method for preparation of electrode material with high performance and low-cost in bioelectrochemical system.
\end{abstract}

\section{Introduction}

Microbial bioelectrochemical system, such as microbial fuel cells (MFCs), is a promising technology for conversion of chemical energy from organic or inorganic chemicals to other kinds of energy by using electroactive bacteria. The great research interests on MFCs can be attributed to their ecofriendly and sustainable characteristics [1], which possess double functions of waste removal and electricity generation. The promising applications of such electrochemical devices involve a broad field including wastewater treatments, hydrogen production [2], remote power sources, desalination [3], and biosensors. The anode in MFCs, which is related to the biofilm growth, plays a crucial role in the performance of MFCs. Various carbon-based materials had been widely employed for anodes in MFCs, such as graphite plate/rod, carbon cloth, carbon felt, carbon paper, reticulated vitrified carbon, and graphite brush, which had been summarized in [4]. Among these, carbon fiber type electrodes were promising anodes for MFCs due to their highly porous architecture. Recently, several new fiber electrodes were developed for anode in MFCs, such as carbon nanotube-textile [5], conductive nanowires network [6], and electrospun carbon fiber mat [7], and had delivered a high current density. However, the direct connection of such electrodes to external circuit remains a big challenge. The fiber mat electrodes, for example, carbon/graphite felts, were bonded with a metal wire or onto a high conductive current collector (e.g., graphite plate or foil) by conductive binders in lab-scale running. Though this method could ensure a uniform and low resistance over the whole mat, only one side of fiber mat was available for microbe growth and thus was impractical for scale-up applications.

Metal materials, such as stainless steel (SS) materials, show excellent mechanical and electrical properties and environmental stability and are of low-cost and easy to be shaped and connected. SS materials had been widely used as cathode [8] or current collector of cathode [9] in MFCs. In one of our previous works, nitrogen-doped carbon nanofibers were self-connected to SSM and used as high performance binderfree cathode in MFCs [10]. The SS materials were also able to be used as anode in MFCs [11], and its performance could be greatly improved by surface modification [12-16]. Anodes with fibers self-connected onto current collectors would be advantageous for MFCs in practical application, because their could combine the macroporous fiber configuration and high conductivity and mechanical strength stainless steel wire together. 
In this study, we proposed a self-connected carbon nanofiber anode design. This design was realized by assembly of thick layer of carbon nanofibers (CNFs) onto stainless steel mesh (SSM) (denoted as CNFs-SSM). The assembly of CNFs on SSM was realized by gas vapor deposition (CVD) process. The anodic performance and biofilm morphology of the CNFs-SSM were studied. The current generated by the CNFsSSM was 200 times higher than the bare SSM under the same experimental conditions.

\section{Materials and Methods}

AISI 304 stainless steel mesh (SSM, 200 mesh) was treated in $1 \mathrm{M} \mathrm{H}_{2} \mathrm{SO}_{4}$ for $4 \mathrm{~h}$ and then was washed with distilled water and dried up. The growth of CNFs onto SSM was conducted in a furnace with a quartz tube in the following steps: SSM was heated to $850^{\circ} \mathrm{C}$ for 30 min under $\mathrm{N}_{2}$ atmosphere with flow rate of $100 \mathrm{~cm}^{3} \cdot \mathrm{min}^{-1}$ and then cooled down to about $750^{\circ} \mathrm{C}$ and at last acetylene gas was fed into the tube with flow rate of $10 \mathrm{~cm}^{3} \cdot \mathrm{min}^{-1}$ for $5 \mathrm{~min}$ and the furnace was left to naturally cool down to room temperature.

The inoculum was secondary biofilms which were selected from domestic wastewater (Qingshan, Nanchang, China) following previous report [17]. Anodes of SSM and CNFs-SSM were cut into pieces with size of $1 \mathrm{~cm} \times 1 \mathrm{~cm}$ and connected with stainless steel wires. The electrochemical test experiments were carried out with computer controlled potentiostat (CHI1040B) which was equipped with eight channels in parallel using one $\mathrm{Ag} / \mathrm{AgCl}$ reference electrode (saturated $\mathrm{KCl}, 0.198 \mathrm{~V}$ versus $\mathrm{SHE}$ ) and one carbon felt counter electrode $\left(4.5 \mathrm{~cm}^{2}\right)$. For the chronoamperometric (CA) measurement, a potential of $+0.2 \mathrm{~V}$ (versus $\mathrm{Ag} / \mathrm{AgCl}$ ) was applied onto the working electrodes and the current was recorded. The current density results were normalized to the geometric area of an electrode. Because both side were available, the area of the electrodes of SSM and CNFs-SSM was $2 \mathrm{~cm}^{2}$.

The cyclic voltammogram (CV) was recorded for turnover and nonturnover conditions. All experimental operations were conducted anaerobically at $35^{\circ} \mathrm{C}$ in $50 \mathrm{mM}$ phosphate buffer solution ( $\mathrm{pH}=7.0$ ) with $10 \mathrm{mM}$ acetate substrate. All of the electrode potentials were versus $\mathrm{Ag} / \mathrm{AgCl}$ (sat. $\mathrm{KCl}$ ) reference electrode. The results were the average values obtained from three independent experiments.

The morphology characterizations were conducted under TESCAN VEGA 3 SEM. Before SEM characterization, the biofilms samples were treated by the following [17]: (a) $5 \mathrm{wt} \%$ glutaric aldehyde solution, (b) a series of ethanol aqueous solutions, and then (c) coating a layer of gold.

\section{Results and Discussion}

The growth of carbon nanotubes or carbon nanofibers on SSM via CVD in the presence or absence of foreign catalysts was widely reported for applications of contamination removal, catalyst supports, electrodes, and so on. The diameters of these materials were in the range of several or tens of nanometers. However, the report of growth of carbon fibers or tubes with diameter over $100 \mathrm{~nm}$ was very rare. As reported in our previous report, continuous biofilms were able to form in the carbon fiber mat anodes with fiber diameter in submicron scale $(0.5-1 \mu \mathrm{m})$ and displayed higher performance than that with larger or smaller fiber diameter [17]. In this paper, carbon nanofibers with diameter in the range of hundreds of nanometers were successfully grown onto SSM by CVD without using any foreign catalysts. As shown in Figures 1(c) and 1(d), the thickness of carbon nanofiber layer grown on the SSM was more than $50 \mu \mathrm{m}$. Detailed observation in Figure 1(d) revealed that the carbon fibers were solid but not hollow and had diameter of about $500 \mathrm{~nm}$. The growth of such big carbon fibers on SSM could be attributed to the rough SSM surface that resulted by $\mathrm{H}_{2} \mathrm{SO}_{4}$ and heat treatment. As indicated by arrows in the inset of Figure 1(a), a number of particles in submicron size were formed on the SSM surface, which was likely to be the catalytic sites for the carbon nanofibers growth. The curved carbon nanofiber formed a macroporous architecture layer which was similar to the architecture of the electrospun carbon fiber mat anode in our previous report [7].

The as-prepared CNFs-SSM was directly tested as anode in MFCs without using any current collector in a halfcell. As shown in Figure 2(a), the bare SSM generated an ultralow current density of $0.0068 \mathrm{~mA} \mathrm{~cm}^{-2}$. After growth of a thick layer of CNFs, the CNFs-SSM anode could generate a maximum current density of $1.28 \mathrm{~mA} \mathrm{~cm}^{-2}$ (Figure 2(b)), which was nearly 200 times as that of acid treated SSM electrode and higher than the graphite rod anode with a current density of $0.9 \mathrm{~mA} \mathrm{~cm}^{-2}$ (Figure 2(c)).

It should be noted that the area of the CNFs-SSM electrode includes the area of SSM matrix and the holes. If the current density normalized to the area of SSM matrix [10], it would be $2.32 \mathrm{~mA} \mathrm{~cm}^{-2}$. The great increase in current density comparing to the bare SSM was brought by the porous CNFs layers, which provided a more compatible surface for biofilms growth. Comparing to the previous result from electrospun carbon nanofiber mat [17], the current density in this study was lower; the possible reason was that carbon fiber layer grown on the SSM was too thin and could not cover the holes in the SSM. The CVs of the CNFs-SSM anode were recorded under the turnover (with substrate) and nonturnover (without substrate) conditions, as shown in Figure 2(d). It confirmed that the current generation was from the metabolism of biofilms on the CNFs-SSM anode.

The morphologies of biofilm on/in the SSM and CNFsSSM were observed by SEM. For the SSM electrode, as shown in Figure 1(b), only a few biofilms were grown at the junctions of two SSM wires, while much less were grown on the SSM wire surface. For the CNFs-SSM, as shown in Figures 1(d)-1(f), the whole electrode was covered by thick biofilms. Figure 1(f) shows that a thick biofilm-CNF with thickness of $10.5 \mu \mathrm{m}$ was formed on the CNFs-SSM anode. It demonstrated that the layer of CNFs on the SSM greatly improved the microbial compatibility of the SSM. The biofilms-CNFs composite film likely ensured a rapid transfer of electrons from microbes to the SSM network. Thus, it can be confirmed that the current boost was brought by the macroporous CNFs layers. 


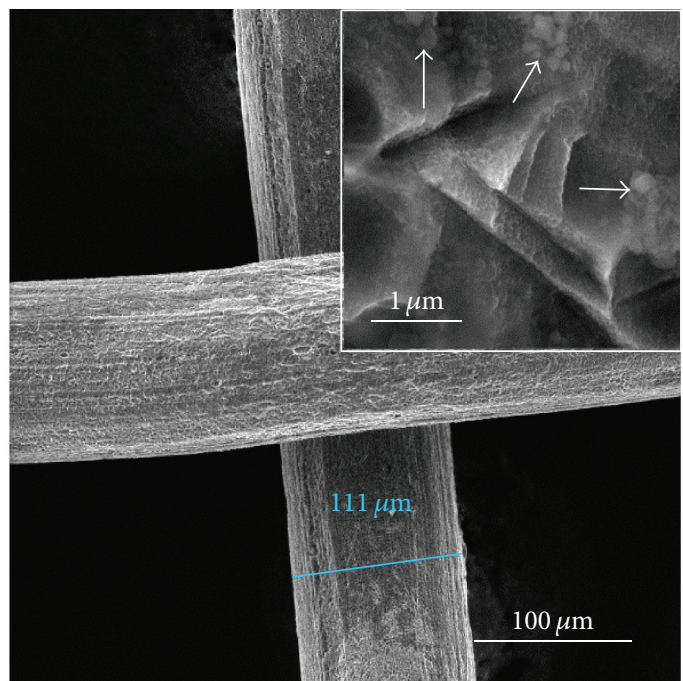

(a)

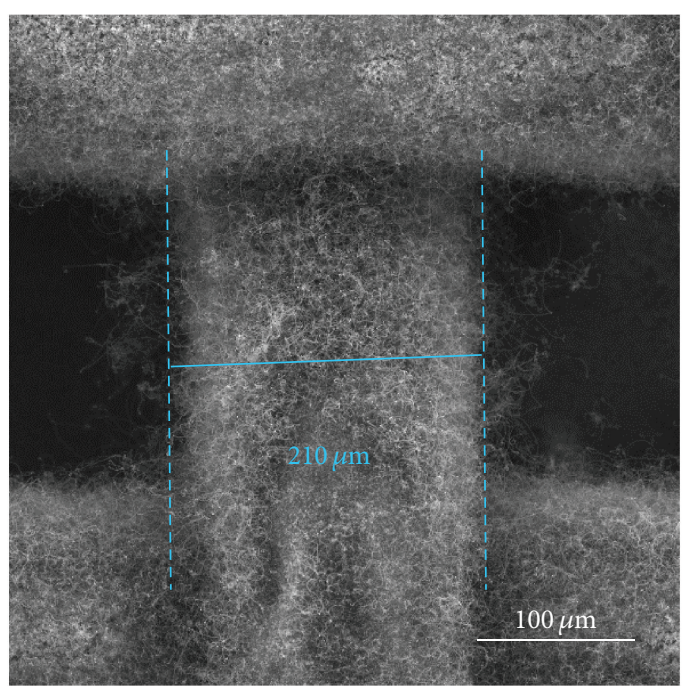

(c)

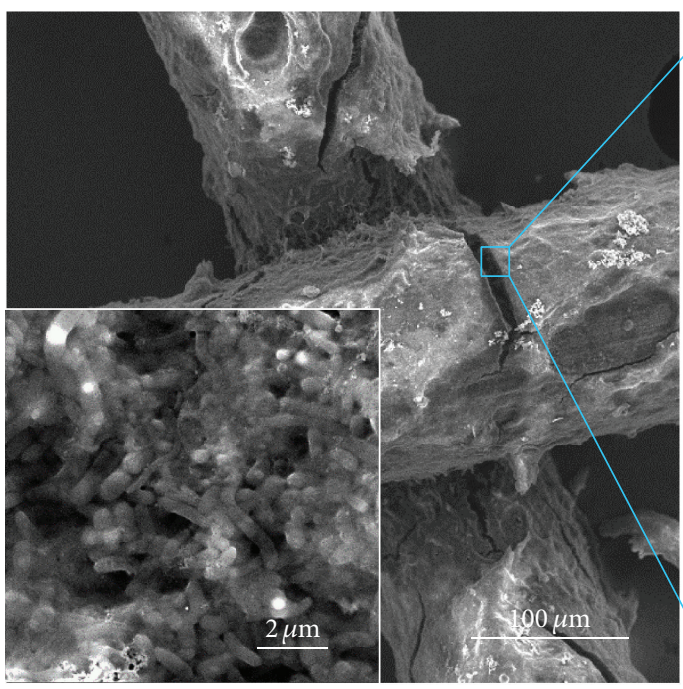

(e)

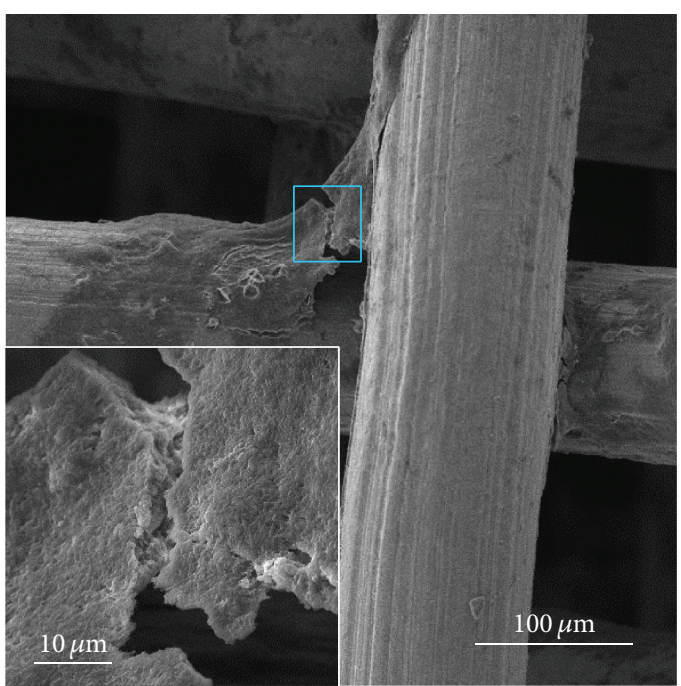

(b)

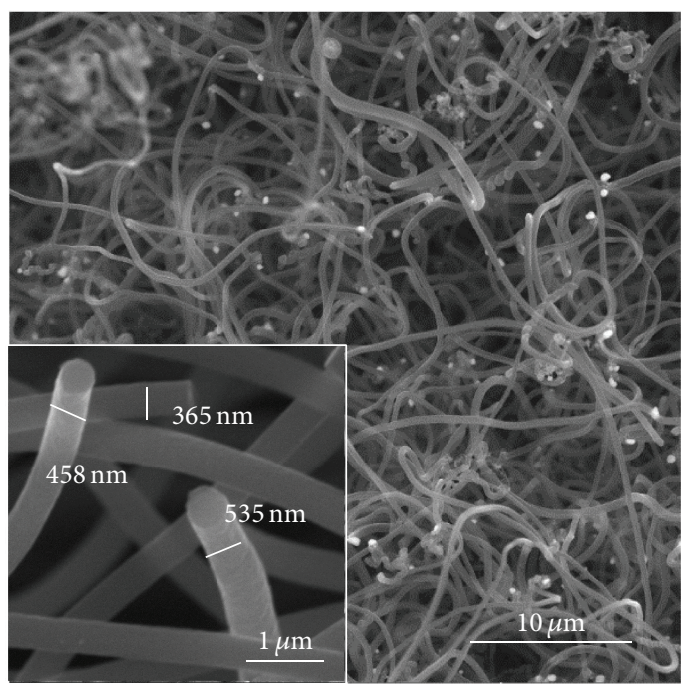

(d)

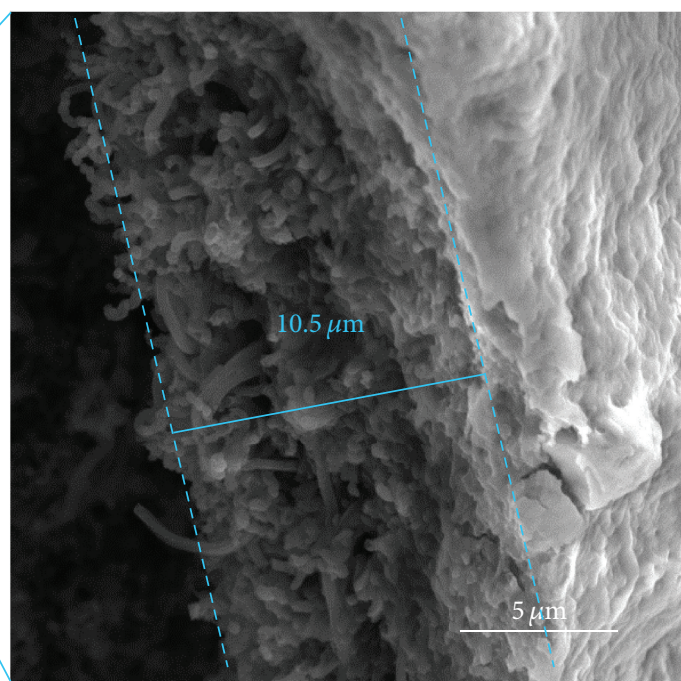

(f)

FIGURE 1: SEM images of (a) SSM, (b) SSM grown with biofilms, (c) and (d) CNFs grown on SSM, (e) CNFs-SSM electrode grown with biofilms, and (f) biofilms on CNFs-SSM showing thickness of $10.5 \mu \mathrm{m}$. Insert of (d) was the cross-sectional image of carbon fibers. Insert of (e) was high resolution of SEM image of biofilms on the CNFs-SSM. 


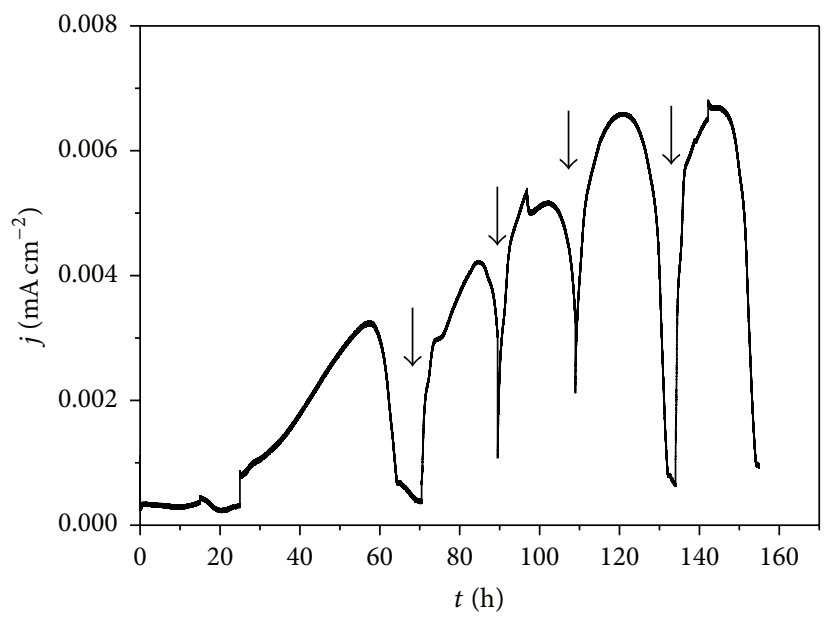

(a)

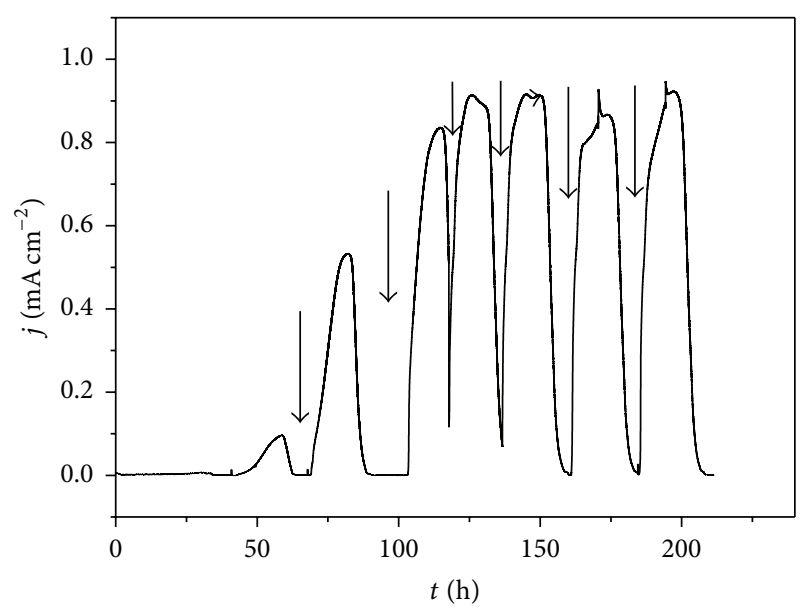

(c)

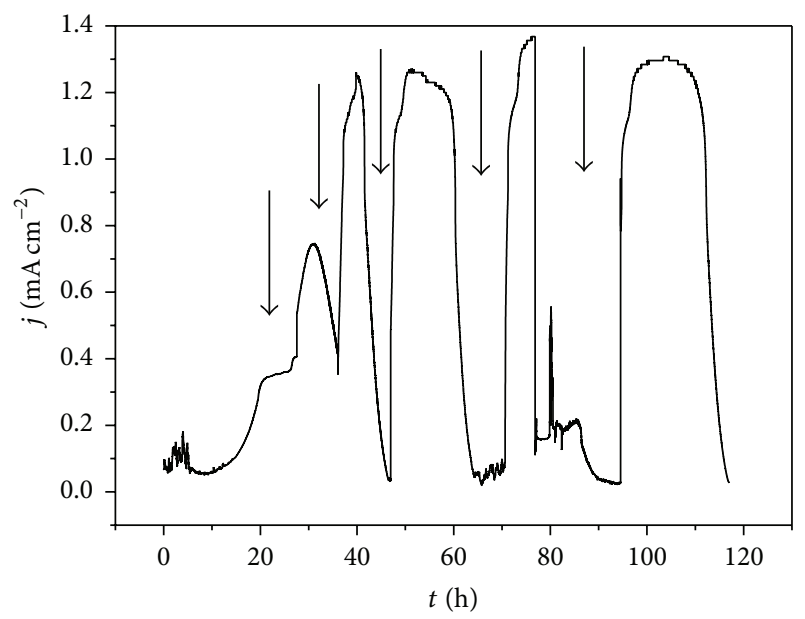

(b)

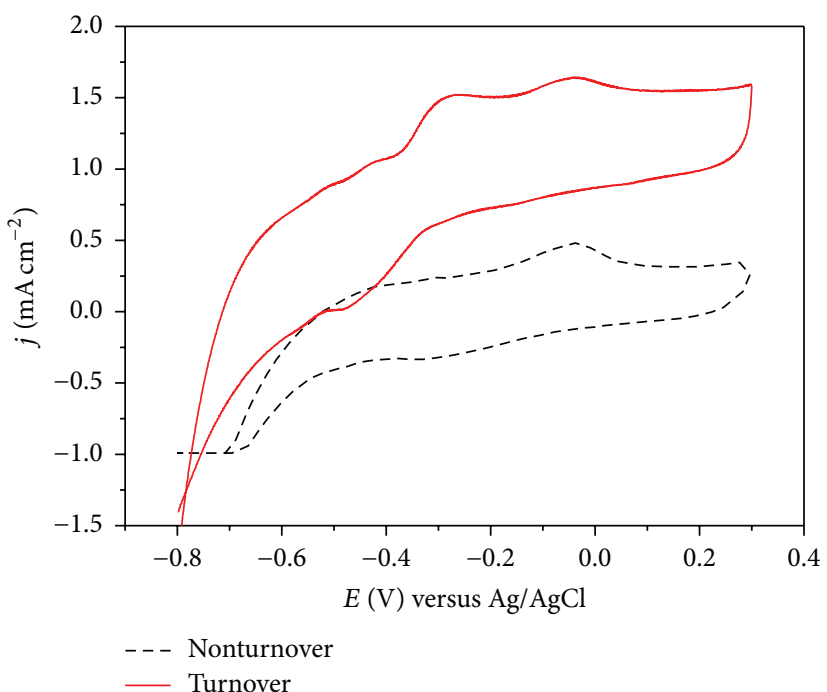

(d)

Figure 2: Current generation of (a) SSM, (b) CNFs-SSM, and (c) graphite rod anodes in a half-cell experiment measured at $35^{\circ} \mathrm{C}$. Arrows represent refresh of media with $10 \mathrm{mM}$ acetate sodium solution. The applying potential was $0 \mathrm{~V}$ versus $\mathrm{Ag} / \mathrm{AgCl}$. (d) Cyclic voltammograms of CNFs-SSM anodes under turnover and nonturnover conditions.

\section{Conclusion}

Self-connected anode of CNFs-SSM was prepared by CVD process without using external catalyst. The CNFs-SSM anode generated 200 times higher current than that of bare SSM. The current boost was attributed to the macroporous submicron carbon fiber layer, which provided a more compatible surface than bare SSM for biofilms propagation. This study provided a simple and promising method for preparation of high performance and low-cost electrode material for bioelectrochemical system like MFCs.

\section{Conflict of Interests}

The authors declare that there is no conflict of interests regarding the publication of this paper.

\section{Acknowledgments}

This research was supported by the National Natural Science Foundation of China (no. 21464008) and the Science and Technology Project of Jiangxi Province (nos. 20121BBE50024 and 21464008).

\section{References}

[1] K. Rabaey and W. Verstraete, "Microbial fuel cells: novel biotechnology for energy generation," Trends in Biotechnology, vol. 23, no. 6, pp. 291-298, 2005.

[2] H. Liu, S. Grot, and B. E. Logan, "Electrochemically assisted microbial production of hydrogen from acetate," Environmental Science \& Technology, vol. 39, no. 11, pp. 4317-4320, 2005.

[3] X. X. Cao, X. Huang, P. Liang et al., "A new method for water desalination using microbial desalination cells," Environmental Science \& Technology, vol. 43, no. 18, pp. 7148-7152, 2009. 
[4] B. E. Logan, Microbial Fuel Cells, John Wiley \& Sons, Hoboken, NJ, USA, 2008.

[5] X. Xie, L. Hu, M. Pasta et al., "Three-dimensional carbon nanotube-textile anode for high-performance microbial fuel cells," Nano Letters, vol. 11, no. 1, pp. 291-296, 2011.

[6] Y. Zhao, K. Watanabe, R. Nakamura et al., "Three-dimensional conductive nanowire networks for maximizing anode performance in microbial fuel cells," Chemistry - A European Journal, vol. 16, no. 17, pp. 4982-4985, 2010.

[7] S. L. Chen, H. Q. Hou, F. Harnisch et al., "Electrospun and solution blown three-dimensional carbon fiber nonwovens for application as electrodes in microbial fuel cells," Energy \& Environmental Science, vol. 4, no. 4, pp. 1417-1421, 2011.

[8] D. F. Call, M. D. Merrill, and B. E. Logan, "High surface area stainless steel brushes as cathodes in microbial electrolysis cells," Environmental Science \& Technology, vol. 43, no. 6, pp. 21792183, 2009.

[9] C. Dumas, R. Basseguy, and A. Bergel, "Microbial electrocatalysis with Geobacter sulfurreducens biofilm on stainless steel cathodes," Electrochimica Acta, vol. 53, no. 5, pp. 2494-2500, 2008.

[10] S. Chen, Y. Chen, G. He, S. He, U. Schröder, and H. Hou, "Stainless steel mesh supported nitrogen-doped carbon nanofibers for binder-free cathode in microbial fuel cells," Biosensors and Bioelectronics, vol. 34, no. 1, pp. 282-285, 2012.

[11] C. Dumas, A. Mollica, D. Féron, R. Basséguy, L. Etcheverry, and A. Bergel, "Marine microbial fuel cell: use of stainless steel electrodes as anode and cathode materials," Electrochimica Acta, vol. 53, no. 2, pp. 468-473, 2007.

[12] S. Zheng, F. Yang, S. Chen et al., "Binder-free carbon black/ stainless steel mesh composite electrode for high-performance anode in microbial fuel cells," Journal of Power Sources, vol. 284, pp. 252-257, 2015.

[13] D. Pocaznoi, A. Calmet, L. Etcheverry, B. Erable, and A. Bergel, "Stainless steel is a promising electrode material for anodes of microbial fuel cells," Energy \& Environmental Science, vol. 5, no. 11, pp. 9645-9652, 2012.

[14] J. L. Lamp, J. S. Guest, S. Naha et al., "Flame synthesis of carbon nanostructures on stainless steel anodes for use in microbial fuel cells," Journal of Power Sources, vol. 196, no. 14, pp. 5829-5834, 2011.

[15] K. Guo, A. H. Soeriyadi, H. Feng et al., "Heat-treated stainless steel felt as scalable anode material for bioelectrochemical systems," Bioresource Technology, vol. 195, pp. 46-50, 2015.

[16] J. Hou, Z. Liu, S. Yang, and Y. Zhou, “Three-dimensional macroporous anodes based on stainless steel fiber felt for high-performance microbial fuel cells," Journal of Power Sources, vol. 258, pp. 204-209, 2014.

[17] G. He, Y. Gu, S. He, U. Schröder, S. Chen, and H. Hou, "Effect of fiber diameter on the behavior of biofilm and anodic performance of fiber electrodes in microbial fuel cells," Bioresource Technology, vol. 102, no. 22, pp. 10763-10766, 2011. 

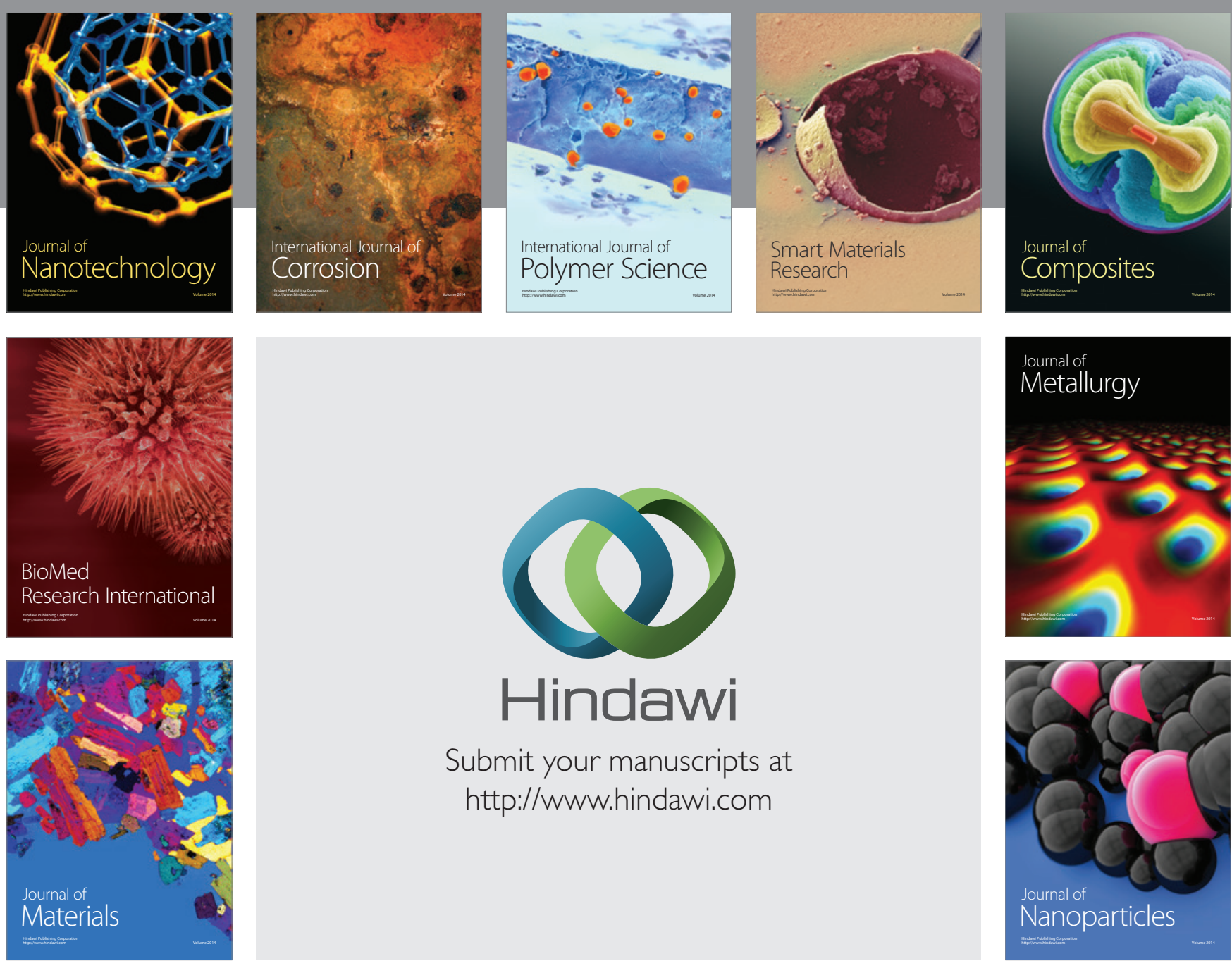

\section{Hindawi}

Submit your manuscripts at

http://www.hindawi.com

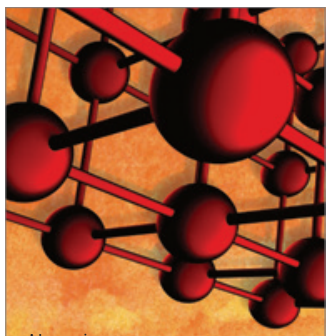

Materials Science and Engineering
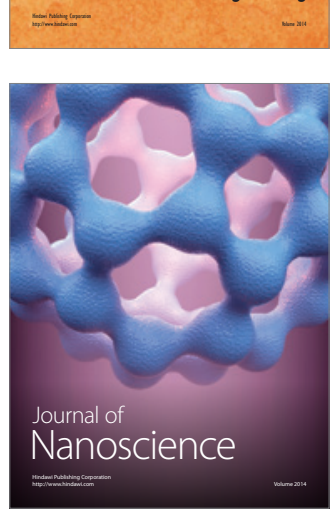
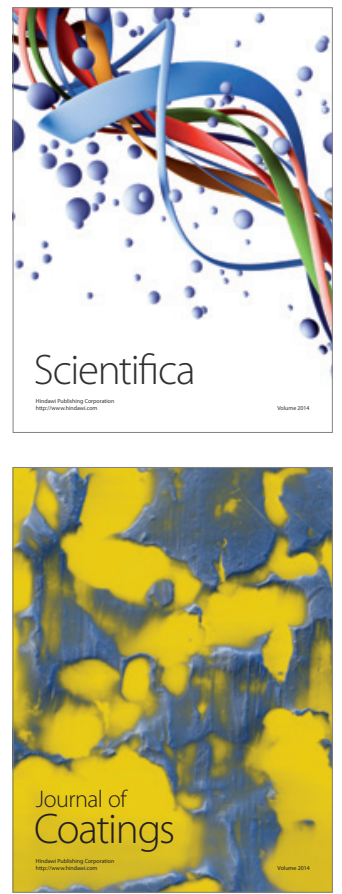
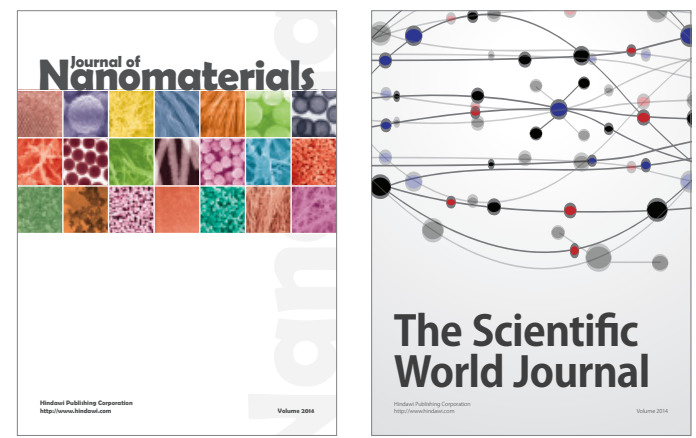

The Scientific World Journal
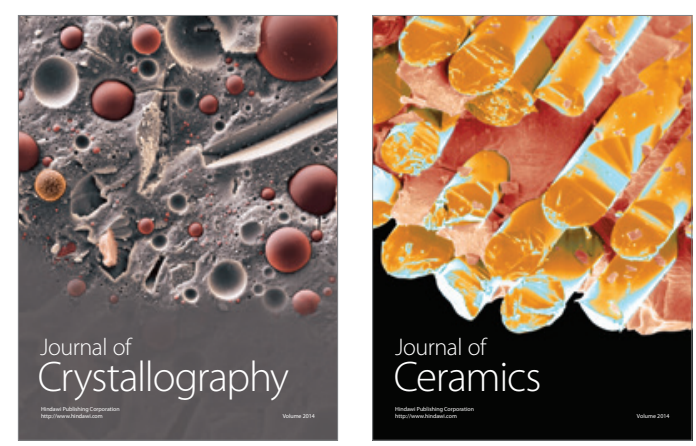
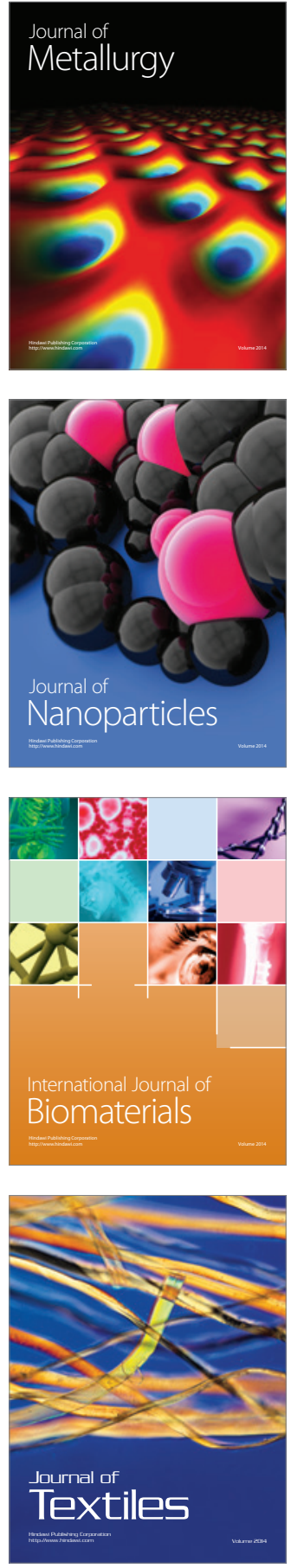